\title{
Serum Bile Acid Profiles Improve Clinical Prediction of Nonalcoholic Fatty Liver in T2DM patients
}

Tao $\mathrm{Wu}^{1,2}$, Ming Yang ${ }^{1}$, Hanchen $\mathrm{Xu}^{1}$, Lei Wang ${ }^{1}$, Huafeng $\mathrm{Wei}^{1}$, Guang $\mathrm{Ji}^{1 *}$

${ }^{1}$ Institute of Digestive Disease, Longhua Hospital, Shanghai University of Traditional Chinese Medicine, Shanghai, China.

${ }^{2}$ Institute of Interdisciplinary Integrative Medicine Research, Shanghai University of Traditional Chinese Medicine, Shanghai, China.

* Correspondence: Guang Ji, PhD, Institute of Digestive Disease, Longhua Hospital, Shanghai University of Traditional Chinese Medicine,

South Wanping Road 725, Shanghai, China. Tel: +86-21-64286261 Fax: +86-21-64286261; E-mail address: jiliver@ vip.sina.com. 
Tables and Figures in Main manuscript and Supporting Information

\begin{tabular}{llll}
\hline Information & Exploration cohort & Validation cohort & Both \\
\hline Clinical information & Figure S1, Table S4 & Figure S4, Figure S5, Table S5 & Table S1 \\
Serum BAs & Table S3 & & Table 2, Figure S2 \\
AUC & Figure 1, Figure 2A & Figure S6 & \\
OPLS-DA analysis & Figure 2B & Figure S5 & Table S2, Figure S3 \\
Spearman correlations & Figure 3A & Figure 3B & Table 3 \\
Decision curve analysis & & & Figure 4 \\
Schematic illustration & & & \\
\hline
\end{tabular}

Table of Contents of Supporting Information

Figure S1. Major components of bile acid profiles in serum from T2DM patients without NAFL and T2DM patients with NAFL as analyzed by

UPLC-TQMS in exploration cohort. 
Figure S2. Receiver-Operator Characteristic curves of five models based on different predictor sets for exploration and validation cohort.

Figure S3. Spearman correlations between the 5 NAFL-associated bile acids and clinical indices that included in exploration and validation cohort.

Figure S4. Major components of bile acid profiles in serum from T2DM patients without NAFL and T2DM patients with NAFL as analyzed by UPLC-TQMS in validation cohort.

Figure S5. Serum concentrations and Spearman correlations among the 5 NAFL-associated BAs in T2DM patients without NAFL and T2DM patients with NAFL as analyzed by UPLC-TQMS in validation cohort.

Figure S6. OPLS-DA analysis of 24 serum BAs from T2DM patients with and without NAFL in validation cohort.

Table S1. Serum bile acid profiles of T2DM patients without NAFL and T2DM patients with NAFL in exploration and validation cohort.

Table S2. Spearman correlations between the five NAFL-associated bile acids and clinical indicators that included in exploration and validation 
cohort.

Table S3. Parameters of 5 models based on five NAFL-associated bile acids and clinical indicators in validation cohort.

Table S4. Serum bile acid Profiles of T2DM male and female patients with or without NAFL in exploration cohort.

Table S5. Serum bile acid Profiles of T2DM male and female patients with or without NAFL in validation cohort. 
(A)

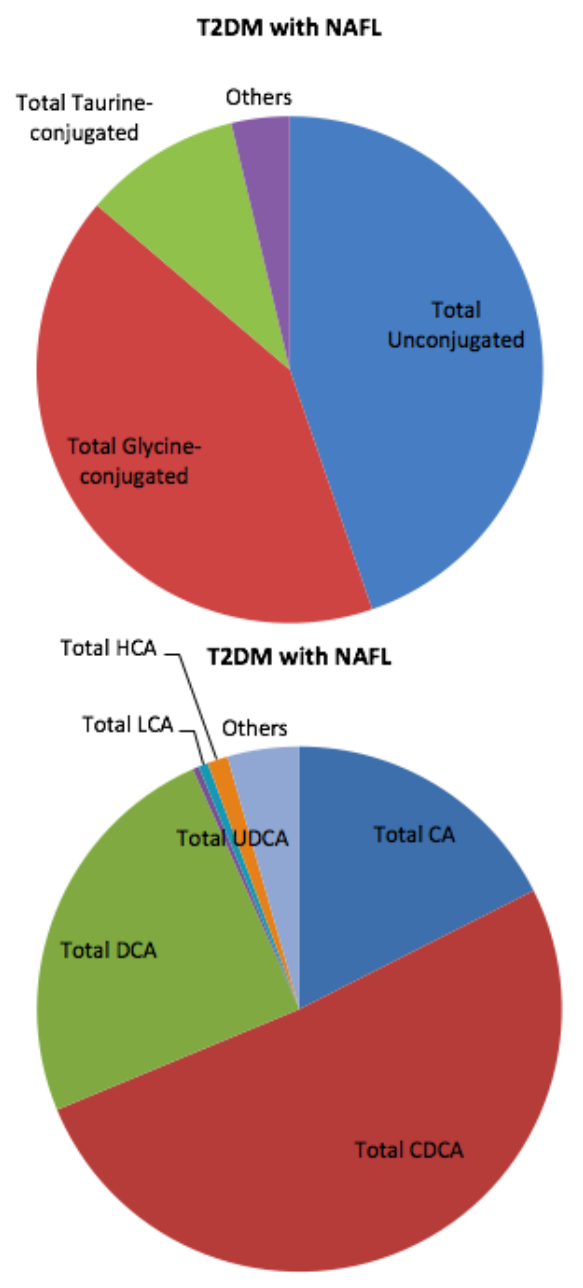

T2DM without NAFL
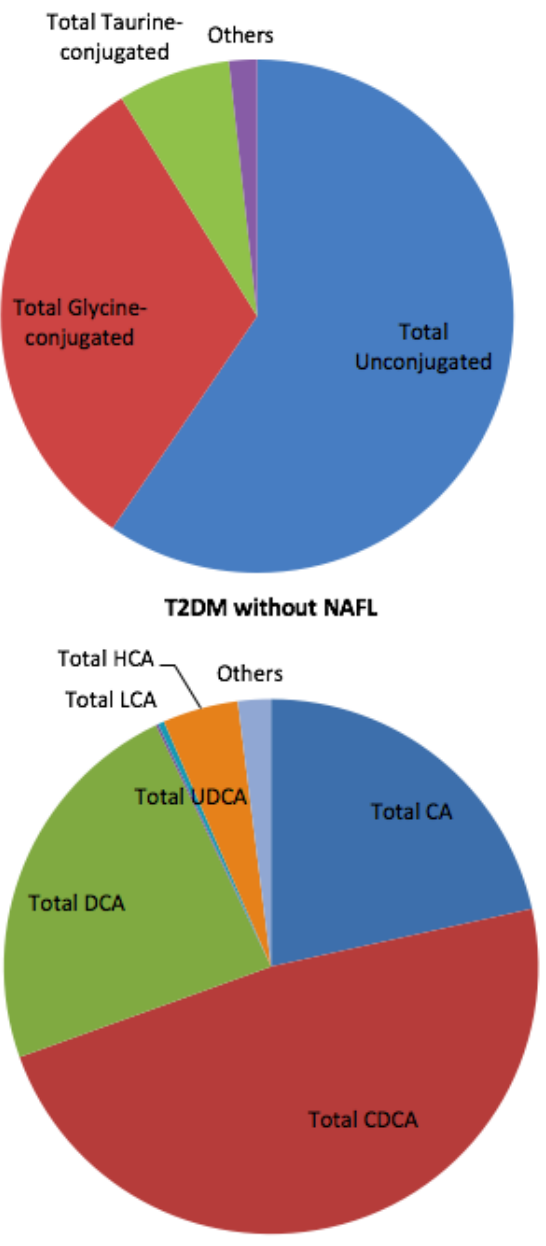
(C)

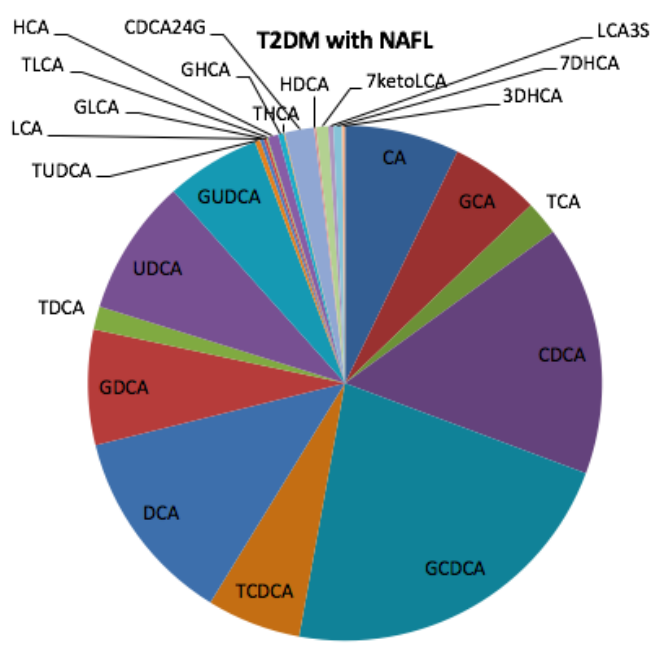

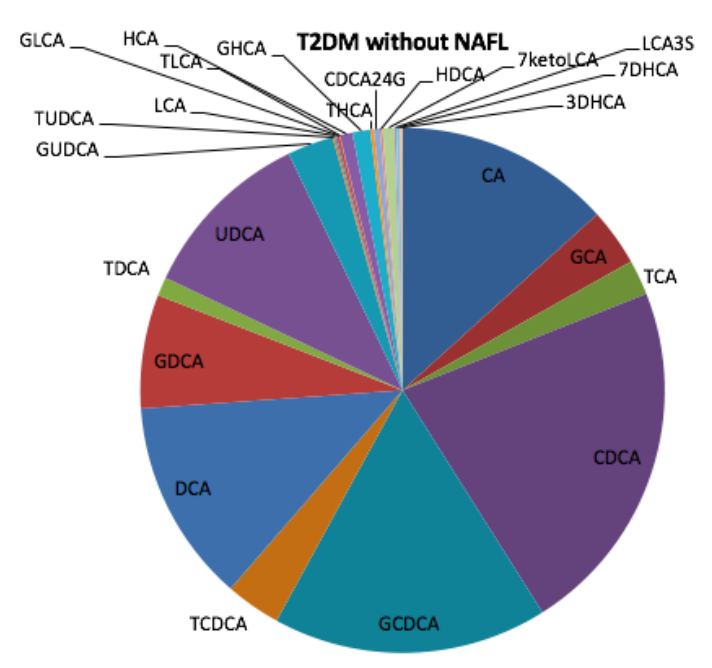

Figure S1. Major components of bile acid profiles in serum from from T2DM patients with or without NAFL analyzed by UPLC-TQMS

in exploration cohort. (A): total BA, unconjugated, glycine-conjugated, taurine-conjugated BAs. (B): total CA, CDCA, DCA, UDCA, LCA,

HCA and others. (C): total 24 bile acids. Abbreviations: T2DM, type 2 diabetes mellitus; NAFL, nonalcoholic fatty liver; TBA, Total bile acids;

CA, cholic acid; GCA, glycocholic acid; TCA, taurocholic acid; CDCA, chenodeoxycholic acid; GCDCA, glycochenodeoxycholic acid;

TCDCA, taurochenodeoxycholic acid; DCA, deoxycholic acid; GDCA, glycodeoxycholic acid; TDCA, taurodeoxycholic acid; UDCA, 
ursodeoxycholic acid; GUDCA, glycoursodeoxycholic acid; TUDCA, tauroursodeoxycholic acid; LCA, lithocholic acid; GLCA, glycolithocholic acid; TLCA, taurolithocholic acid; HCA, hyocholic acid; GHCA, glycohyocholic acid; THCA, taurohyocholic acid; HDCA, hyodeoxycholic acid; GHDCA, glycohyodeoxycholic acid; THDCA, taurohyodeoxycholic acid; DHCA, dehydrocholic acid; GDHCA, glycodehydrocholic acid; TDHCA, taurodehydrocholic acid; 7-KLCA, 7-ketolithocholicacid; LCA-3S, lithocholic acid-3 sulfate; 3-DHCA, 3-dehydrocholic acid; 7-DHCA, 7-dehydrocholic acid; CDCA-24G, acyl CDCA-24 glucuronide. 

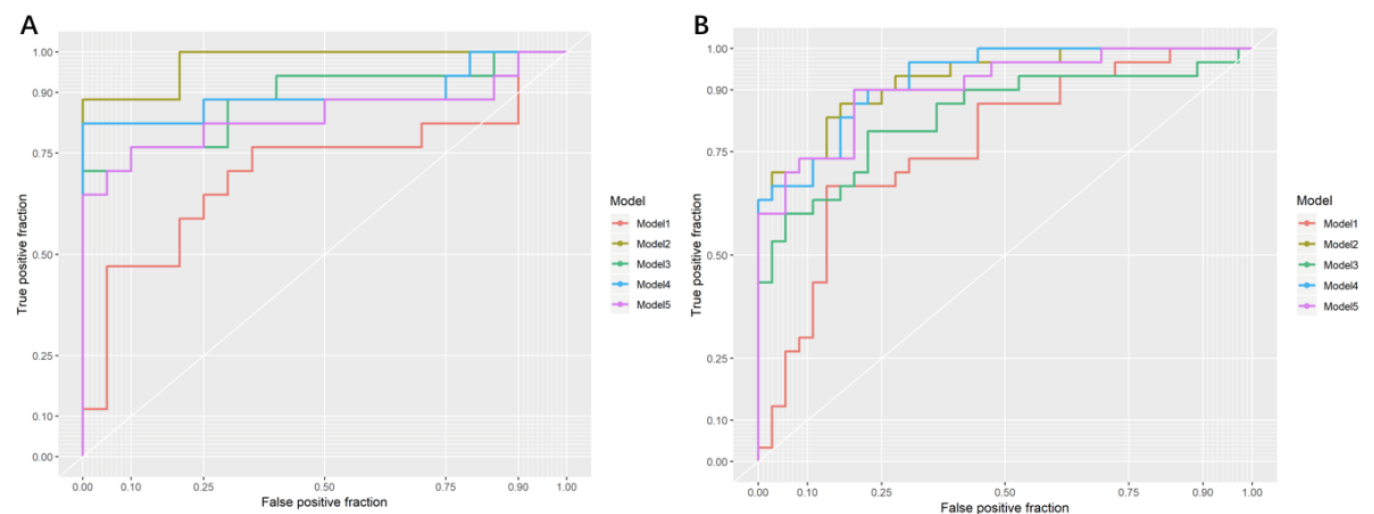

Figure S2. Receiver-Operator Characteristic curves of five models based on different predictor sets for exploration and validation

cohort. (A): exploration cohort; (B): Validation cohort. Model 1, clinical factors, including age, sex, BMI, WHR, FPG, 2h PG, ALT, TG, HDL

and LDL; Model 2, all metabolites; Model 3, biomarker metabolites; Model 4, clinical factors plus all metabolites ; Model 5, clinical factors plus

biomarker metabolites. 

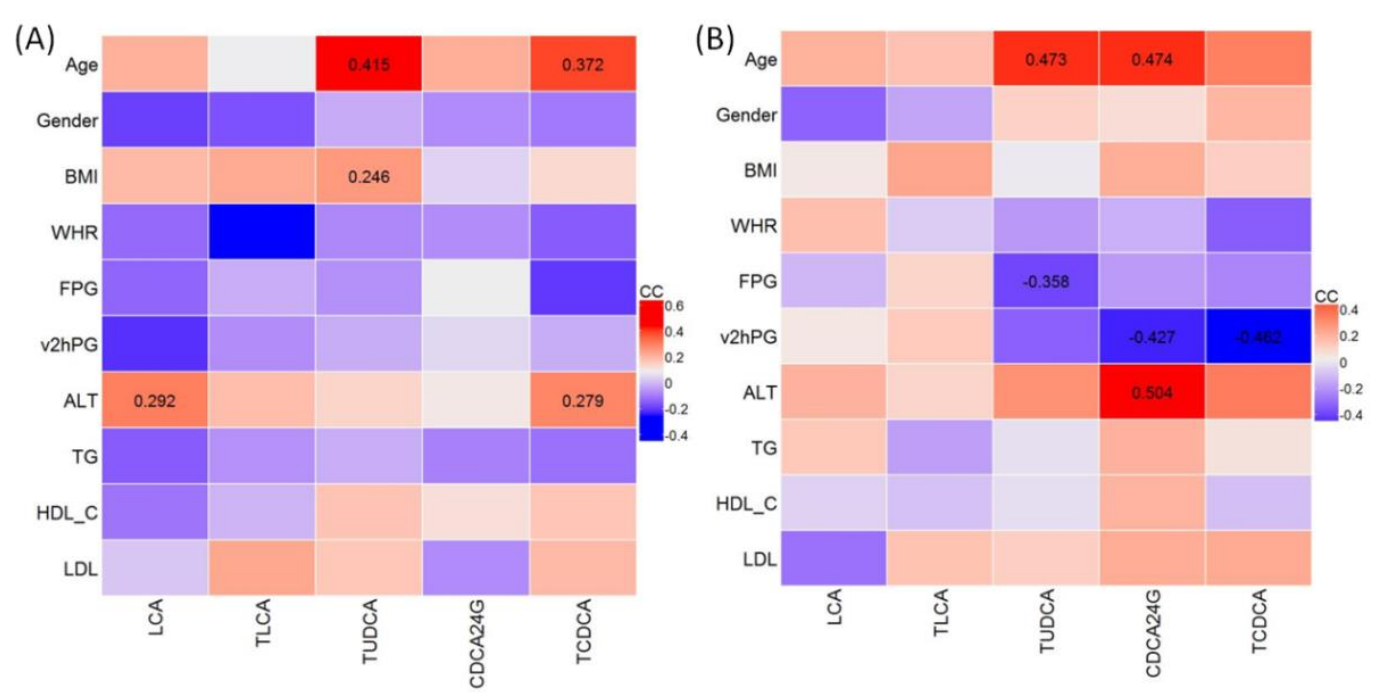

Figure S3. Spearman correlations between the 5 NAFL-associated bile acids and clinical indices that included in exploration and

validation cohort. (A): exploration cohort; (B): validation cohort. The displayed numbers of the correlation in the figures means significant

correlation coefficients between bile acids and clinical indices. BMI, body mass index; WHR, waist-hip ratio; FPG, fasting plasma glucose; 2 h

PG, 2 h plasma glucose; ALT, alanine aminotransferase; TG, triglyceride; HDL, high-density lipoprotein; LDL, low-density lipoprotein; LCA,

lithocholic acid; TLCA, taurolithocholic acid; TUDCA, tauroursodeoxycholic acid; CDCA-24G, acyl CDCA-24 glucuronide; TCDCA, 
taurochenodeoxycholic acid. 
(A)

T2DM with NAFL

Total Taurine-

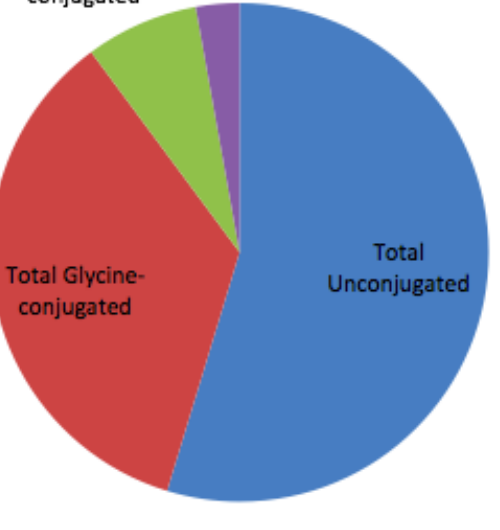

(B)

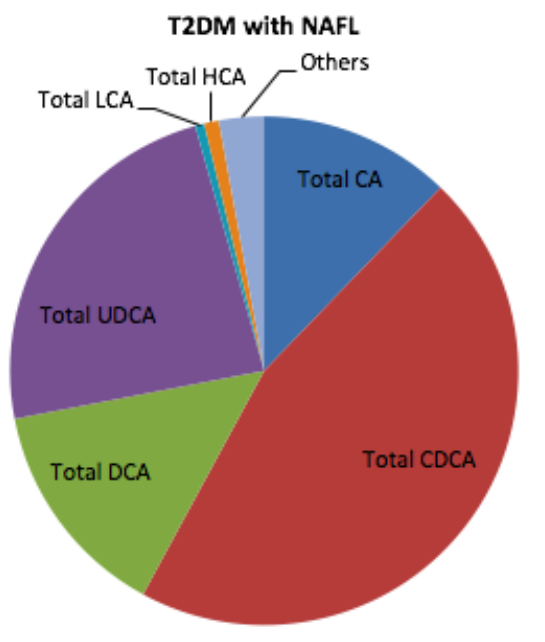

T2DM without NAFL

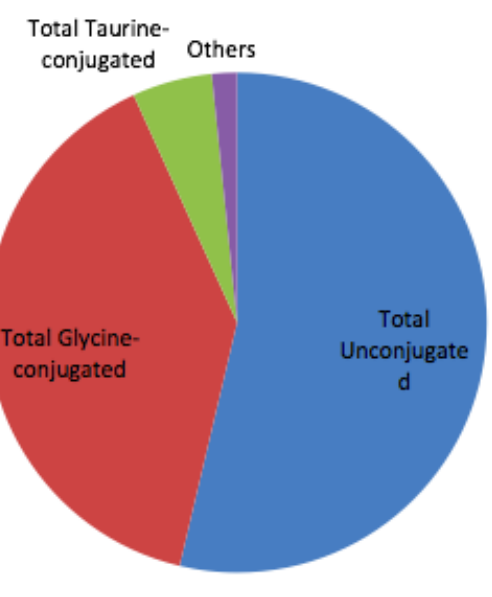

T2DM without NAFL

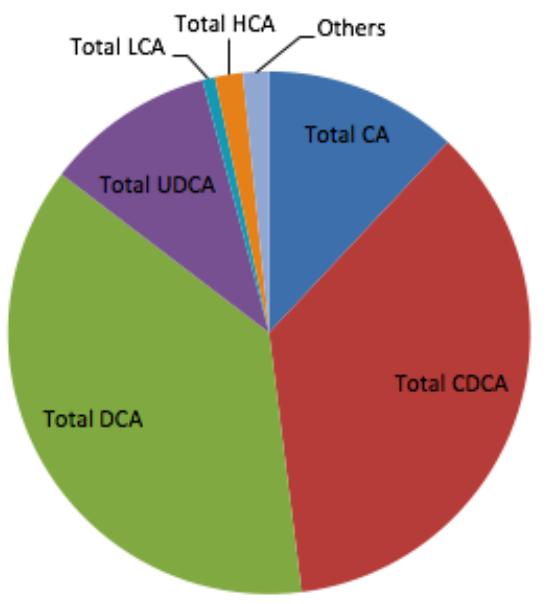


(C)

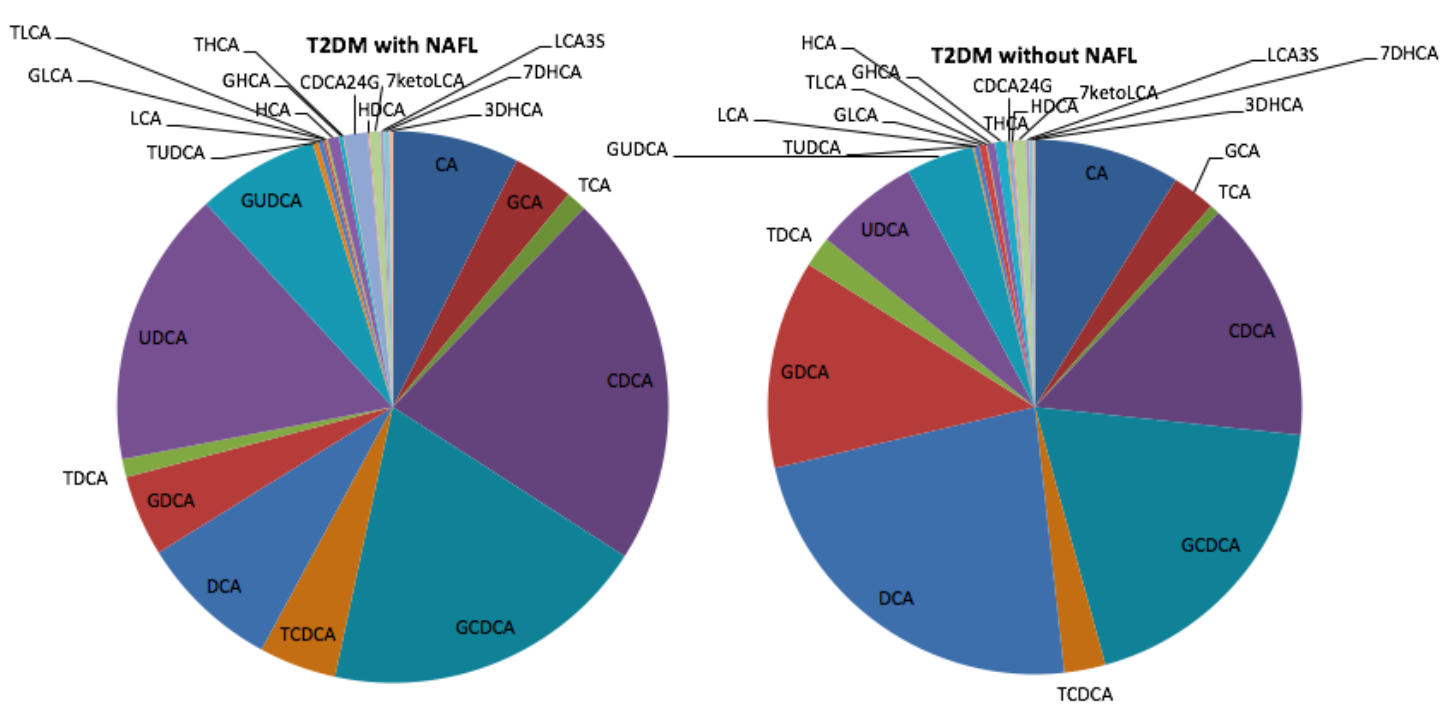

Figure S4. Major components of bile acid profiles in serum from T2DM patients with or without NAFL analyzed by UPLC-TQMS in

validation cohort. (A): total BA, unconjugated, glycine-conjugated, taurine-conjugated BAs. (B): total CA, CDCA, DCA, UDCA, LCA, HCA

and others. (C): total 24 bile acids. Abbreviations: T2DM, type 2 diabetes mellitus; NAFL, nonalcoholic fatty liver; TBA, Total bile acids; CA,

cholic acid; GCA, glycocholic acid; TCA, taurocholic acid; CDCA, chenodeoxycholic acid; GCDCA, glycochenodeoxycholic acid; TCDCA, 
taurochenodeoxycholic acid; DCA, deoxycholic acid; GDCA, glycodeoxycholic acid; TDCA, taurodeoxycholic acid; UDCA, ursodeoxycholic acid; GUDCA, glycoursodeoxycholic acid; TUDCA, tauroursodeoxycholic acid; LCA, lithocholic acid; GLCA, glycolithocholic acid; TLCA, taurolithocholic acid; HCA, hyocholic acid; GHCA, glycohyocholic acid; THCA, taurohyocholic acid; HDCA, hyodeoxycholic acid; GHDCA, glycohyodeoxycholic acid; THDCA, taurohyodeoxycholic acid; DHCA, dehydrocholic acid; GDHCA, glycodehydrocholic acid; TDHCA, taurodehydrocholic acid; 7-KLCA, 7-ketolithocholicacid; LCA-3S, lithocholic acid-3 sulfate; 3-DHCA, 3-dehydrocholic acid; 7-DHCA, 7-dehydrocholic acid; CDCA-24G, acyl CDCA-24 glucuronide. 


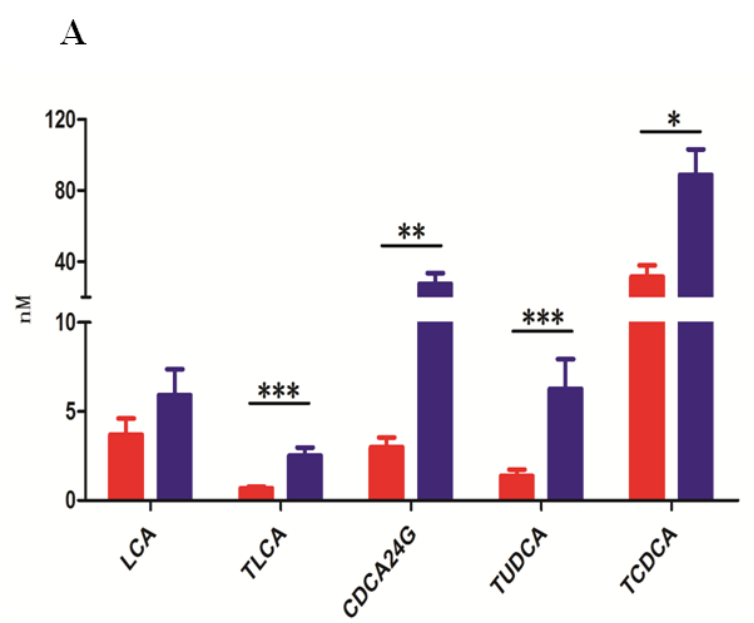

B

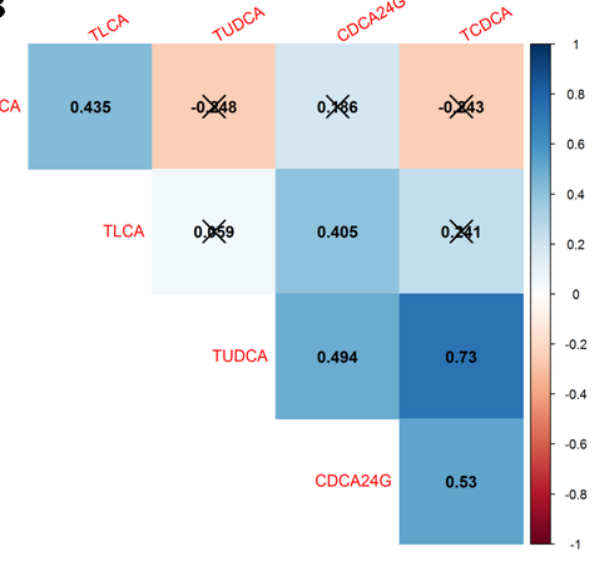

Figure S5. Serum concentrations and Spearman correlations among the 5 NAFL-associated BAs in T2DM patients with or without

NAFL analyzed by UPLC-TQMS in validation cohort. (A): * $p<0.05, * * p<0.01, * * * p<0.001$, compared with T2DM patients without NAFL.

(B): Strong correlations $(P<0.05)$ were observed between LCA and TLCA $(\rho=0.435)$; TLCA and CDCA24G ( $\rho=0.405)$; TUDCA and CDCA24G ( $\rho=0.494)$; TUDCA and TCDCA $(\rho=0.73)$; CDCA24G and TCDCA $(\rho=0.53)$. " $\boldsymbol{*}$ ' means no significant correlation between the two

BAs, ie $P>0.05$. No “ $*$ ' means significant correlation between the two BAs, ie $P<0.05$. 
T2DM, type 2 diabetes mellitus; NAFL, nonalcoholic fatty liver; UPLC-TQMS, ultra-performance liquid chromatography-triple quadrupole mass spectrometry; LCA, lithocholic acid; TLCA, taurolithocholic acid; TUDCA, tauroursodeoxycholic acid; CDCA-24G, acyl CDCA-24 glucuronide; TCDCA, taurochenodeoxycholic acid.

represents T2DM with NAFLD, $\quad$ represents T2DM without NAFLD. 


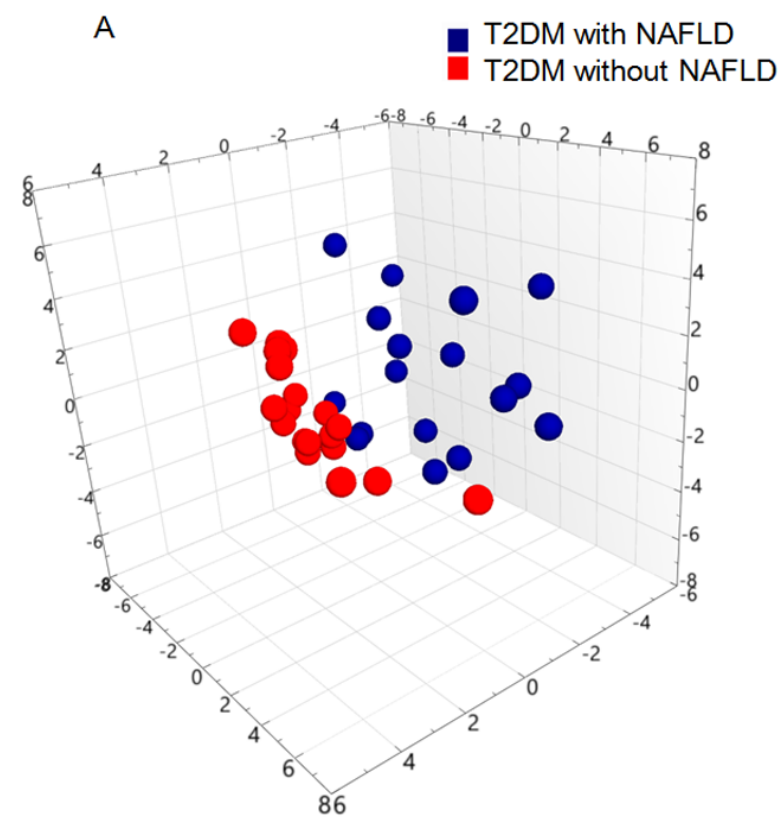

B

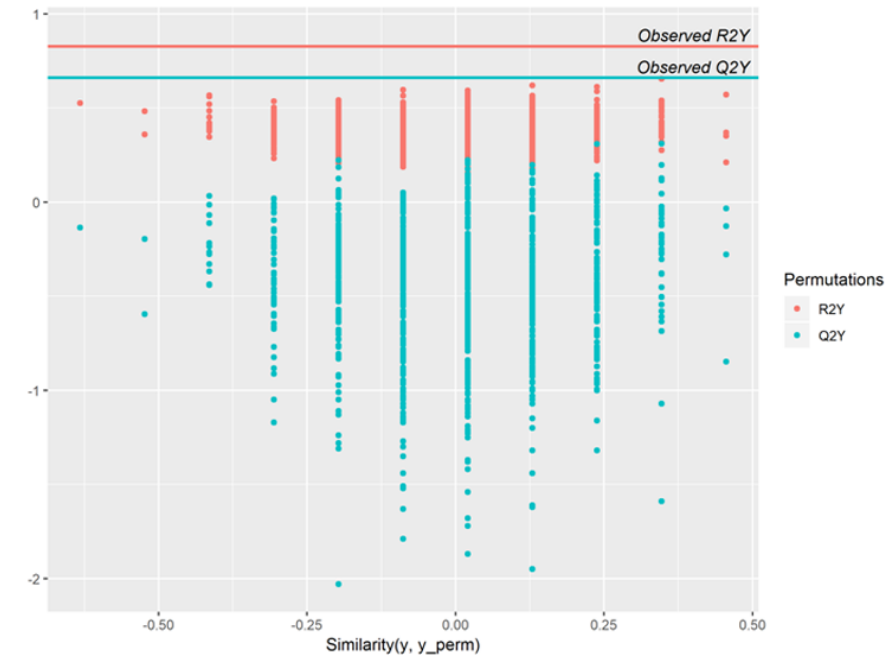

Figure S6. OPLS-DA analysis of 24 serum BAs from T2DM patients with and without NAFL in validation cohort. (A), 3D OPLS-DA

Plots, R2X=0.552, R2Y=0.828, Q2Y=0.660; (B), Permutation analysis. T2DM, type 2 diabetes mellitus; NAFL, nonalcoholic fatty liver;

OPLS-DA, orthogonal projections to latent structures discriminant analysis. 
Tables

Table S1. Serum Bile Acid Profiles of T2DM patients with or without NAFL in exploration and validation cohort.

\begin{tabular}{|c|c|c|c|c|c|}
\hline \multirow[t]{3}{*}{ Variables } & \multicolumn{3}{|l|}{ Exploration cohort } & \multicolumn{2}{|l|}{ Validation cohort } \\
\hline & T2DM patients without & T2DM patients with & $P$ & T2DM patients without & T2DM patients with \\
\hline & $\operatorname{NAFL}(n=36)$ & NAFL(n=30) & & NAFL(n=20) & NAFL(n=17) \\
\hline \multirow[t]{2}{*}{ Total Unconjugated } & $956.45(532.625-2353.7)$ & $1275.15(684.975-1919.7$ & & $395.6(215.375-763.2)$ & $1062.6(454.1-1686.6$ \\
\hline & & 75) & & & )$^{*}$ \\
\hline Total & $695.45(407.025-1034.15)$ & $835.65(582.875-1972.45)$ & & $478.65(352.725-592.425)$ & $708.2(326.1-955.5)$ \\
\hline
\end{tabular}




\begin{tabular}{|c|c|c|c|c|}
\hline Glycine-conj & & & & \\
\hline Total & 81.7(38.825-176.9) & $191.15(107.8-394.225)^{* *}$ & 48.7(35.75-88.075) & $115.5(63.4-219)^{* *}$ \\
\hline Taurine-conjl & & & & \\
\hline Total CA & $252.5(111.675-562.425)$ & $352.25(129.825-696.15)$ & 71.95(36.725-163.25) & 179.3(106.1-311.7)* \\
\hline CA & $128.85(30.625-429.6)$ & 79.15(41.425-295.625) & $25.65(6.55-94.15)$ & 73.4(29.1-156.5) \\
\hline GCA & 47.6(23.725-113.075) & 70.6(45.325-206.825) & 28.2(20.175-44.3) & $52.7(24-117.6)$ \\
\hline TCA & 7.8(4.15-33.525) & $20.6(9.05-62.575)^{*}$ & $4.25(3.25-9.275)$ & $18.7(5.9-34.9)^{* * *}$ \\
\hline Total CDCA & $994.35(456.55-1930.625)$ & $1007.15(632.775-1843.2)$ & $391.7(184.475-542.975)$ & $809(597-1223.9)^{* *}$ \\
\hline CDCA & $374.65(100.825-816.85)$ & $363.85(163.225-686.475)$ & 50.55(18.675-180.35) & $304.9(135.8-798.9)^{*}$ \\
\hline
\end{tabular}




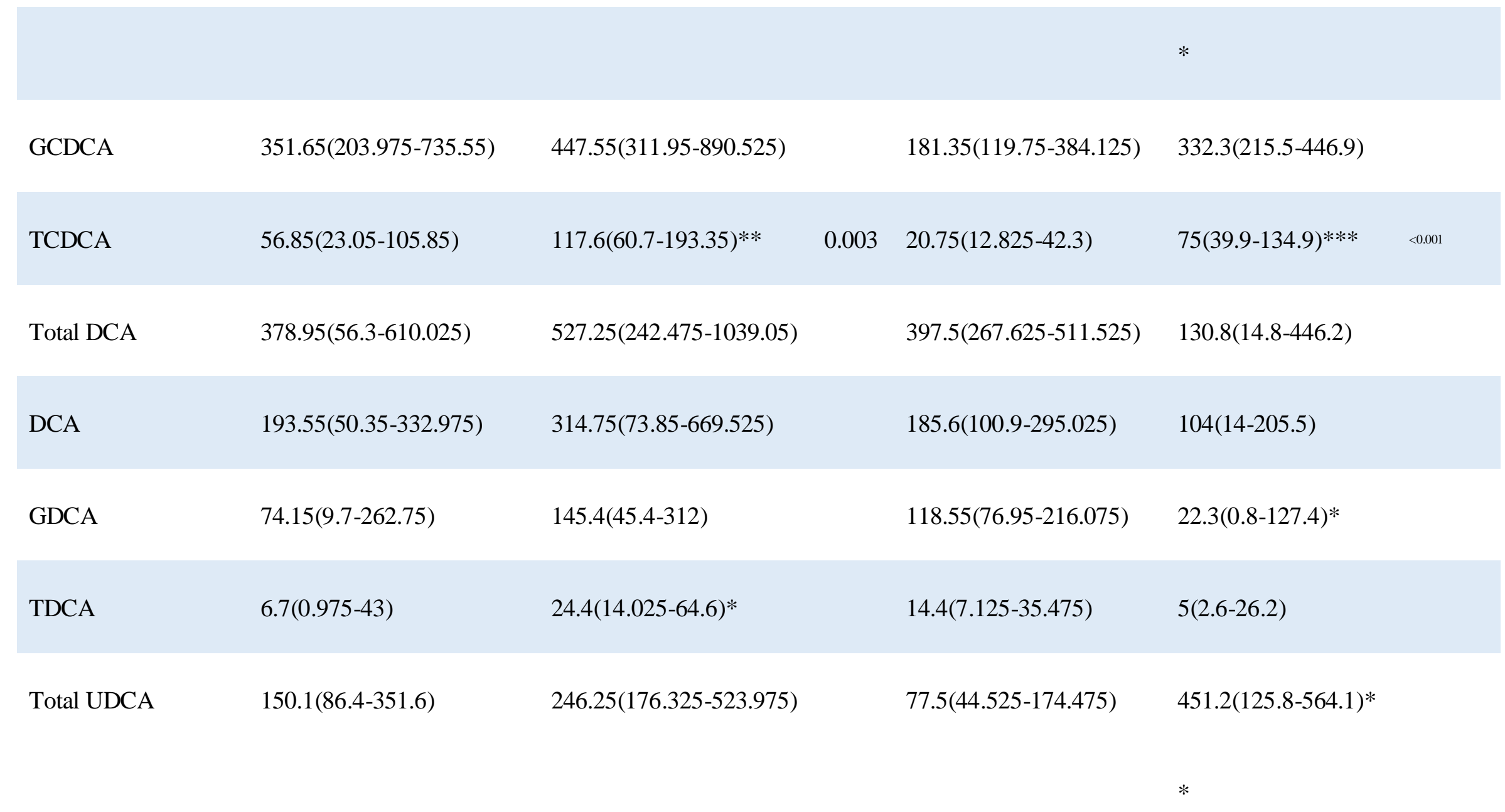




\begin{tabular}{|c|c|c|c|c|c|c|}
\hline UDCA & $100.4(41.125-217.025)$ & 153.95(97.2-296.525) & & $42.1(25.1-77.575)$ & \multicolumn{2}{|l|}{$271.6(43.6-462.2)^{*}$} \\
\hline GUDCA & $51.4(19.5-112.725)$ & $92.3(46.125-278.525)^{*}$ & & $34.6(11.475-86.075)$ & \multicolumn{2}{|l|}{$95.3(48.6-190.4)^{*}$} \\
\hline TUDCA & $1.8(0.675-3.7)$ & $4.55(2.2-8.2)^{* *}$ & 0.009 & $0.9(0.225-1.75)$ & $3.4(2.3-10.2)^{* *}$ & 0.003 \\
\hline Total LCA & $4.65(1.075-10.775)$ & $14.85(2.675-27.7)^{*}$ & & $8(4.125-16)$ & \multicolumn{2}{|l|}{$7.1(1.5-21)$} \\
\hline LCA & $0.25(0-3.525)$ & $7.8(0.825-13.175) * *$ & & $2.15(0-6.7)$ & \multicolumn{2}{|l|}{$4.1(0.1-10.5)$} \\
\hline GLCA & 2.2(0.4-6.975) & $2.9(0-8.5)$ & & $3.75(1.975-7.175)$ & \multicolumn{2}{|l|}{$0(0-3.1)^{*}$} \\
\hline TLCA & $0.6(0.6-0.825)$ & $3.4(1.1-4.15)^{* * *}$ & $<0.00$ & $0.6(0.5-0.8)$ & $3.1(1.1-3.6)^{* *}$ & 0.002 \\
\hline & & & \multicolumn{4}{|l|}{1} \\
\hline Total HCA & $39.65(23.775-57.125)$ & 28.9(16.9-46.65) & & $14.7(12.125-21.675)$ & \multicolumn{2}{|l|}{$15.8(7.8-22.7)$} \\
\hline
\end{tabular}




\begin{tabular}{|c|c|c|c|c|c|c|}
\hline HCA & $14.9(5.075-24.15)$ & $14.6(7.275-25.8)$ & & $3.85(1.175-7.75)$ & $13(3.4-17.1)^{*}$ & \\
\hline GHCA & $6.9(3.95-14.5)$ & $8(3.8-14.3)$ & & $4.9(3.175-8.85)$ & $3.1(1.6-5.6)$ & \\
\hline THCA & $1(0.7-1.6)$ & $1.15(0.3-2.625)$ & & $0.8(0.2-0.925)$ & $0.4(0.3-0.9)$ & \\
\hline \multirow[t]{2}{*}{ CDCA24G } & $4.45(1.8-10.8)$ & $22.35(16-43.625)^{* * *}$ & $<0.00$ & $2.15(1.65-3)$ & $16.9(11.4-28)^{* * *}$ & $<0.001$ \\
\hline & \multicolumn{6}{|c|}{1} \\
\hline HDCA & $0.5(0-2.175)$ & $2.55(0.25-5.15)^{*}$ & & $1.25(0-1.625)$ & $1.2(1.1-2.7)$ & \\
\hline 7ketoLCA & $9.65(3.35-26.275)$ & $11.75(6.95-21.125)$ & & $4.65(2.55-8.575)$ & $8.5(2.6-15.5)$ & \\
\hline LCA3S & $1.95(0.675-6)$ & $7.55(0-14.05)$ & & $2.35(1.25-3.2)$ & $0(0-5)$ & \\
\hline 7DHCA & $3.1(0.825-12.725)$ & 7.1(3.15-12.025) & & $1.35(0.55-2.25)$ & $6(2.3-12)^{* *}$ & \\
\hline
\end{tabular}




$\begin{array}{llll}3 \mathrm{DHCA} & 3.25(1.3-5.35) & 3.45(2.35-7.5) & 0.9(0.225-1.475)\end{array}$

Data are mean $\pm \operatorname{SEM}(\mathrm{nmol} / \mathrm{l}) .{ }^{*} p<0.05, * * p<0.01, * * * p<0.001$, compared with T2DM without NAFLD. TBA, Total bile acids; CA, cholic acid; GCA, glycocholic acid; TCA, taurocholic acid; CDCA, chenodeoxycholic acid; GCDCA, glycochenodeoxycholic acid; TCDCA, taurochenodeoxycholicacid; DCA, deoxycholic acid; GDCA, glycodeoxycholicacid; TDCA, taurodeoxycholic acid; UDCA, ursodeoxycholic acid; GUDCA, glycoursodeoxycholic acid; TUDCA, tauroursodeoxycholic acid; LCA, lithocholicacid; GLCA, glycolithocholic acid; TLCA, taurolithocholicacid; HCA, hyocholic acid; GHCA, glycohyocholic acid; THCA, taurohyocholic acid; HDCA, hyodeoxycholic acid; GHDCA, glycohyodeoxycholicacid; THDCA, taurohyodeoxycholic acid; DHCA, dehydrocholic acid; GDHCA, glycodehydrocholic acid; TDHCA, taurodehydrocholic acid; 7-KLCA, 7-ketolithocholicacid; LCA-3S, lithocholic acid-3 sulfate; 3-DHCA, 3-dehydrocholic acid; 7-DHCA, 7-dehydrocholicacid; CDCA-24G, acyl CDCA-24glucuronide.

Table S2. Spearman correlations between the five NAFL-associated bile acids and clinical indicators that included in exploration and validation 
cohort.

\begin{tabular}{|c|c|c|c|c|c|}
\hline Correlations & LCA & TLCA & TUDCA & CDCA24G & TCDCA \\
\hline Gender & $-0.218^{*}$ & -0.136 & 0.036 & -0.01 & 0.019 \\
\hline Age & $0.194 *$ & 0.078 & $0.387 * * *$ & $0.249 *$ & $0.285^{* *}$ \\
\hline BMI & 0.145 & $0.215^{*}$ & 0.159 & 0.09 & 0.093 \\
\hline WHR & -0.018 & -0.174 & -0.082 & -0.051 & -0.173 \\
\hline FPG & -0.099 & 0.041 & -0.139 & 0.038 & -0.187 \\
\hline $2 \mathrm{hPG}$ & -0.114 & 0.008 & -0.099 & -0.094 & -0.132 \\
\hline TG & -0.041 & -0.087 & -0.006 & 0.011 & -0.034 \\
\hline
\end{tabular}




\begin{tabular}{|c|c|c|c|c|c|}
\hline HDL-C & -0.066 & -0.023 & 0.114 & 0.145 & 0.101 \\
\hline ALT & $0.242 *$ & 0.137 & $0.197 *$ & $0.257 * *$ & $0.303 * *$ \\
\hline LDL & -0.056 & $0.194 *$ & 0.177 & 0.02 & $0.224 *$ \\
\hline 7ketoLCA & -0.173 & $-0.237 *$ & $0.42 * * *$ & $0.324 * * *$ & $0.286^{* *}$ \\
\hline UDCA & $-0.224^{*}$ & -0.097 & $0.679 * * *$ & $0.417 * * *$ & $0.326 * * *$ \\
\hline HDCA & $0.619 * * *$ & $0.419 * * *$ & $-0.202 *$ & $0.202^{*}$ & 0.062 \\
\hline CDCA & $-0.245^{*}$ & $-0.224^{*}$ & $0.321 * * *$ & $0.362 * * *$ & $0.315^{* *}$ \\
\hline DCA & $0.535^{* * *}$ & $0.354 * * *$ & $-0.197 *$ & -0.013 & -0.093 \\
\hline 7DHCA & $-0.216^{*}$ & -0.125 & $0.293 * *$ & $0.364 * * *$ & $0.196^{*}$ \\
\hline
\end{tabular}




\begin{tabular}{|c|c|c|c|c|c|}
\hline 3DHCA & $-0.258 * *$ & -0.119 & $0.299 * *$ & $0.383 * * *$ & $0.316 * *$ \\
\hline $\mathrm{HCA}$ & -0.133 & $-0.202 *$ & $0.303^{* *}$ & $0.395^{* * *}$ & $0.255^{* *}$ \\
\hline $\mathrm{CA}$ & -0.19 & $-0.295 * *$ & 0.156 & $0.259 * *$ & $0.23 *$ \\
\hline GLCA & $0.488 * * *$ & $0.451 * * *$ & $-0.247 *$ & -0.174 & -0.003 \\
\hline GUDCA & -0.161 & -0.017 & $0.902 * * *$ & $0.348 * * *$ & $0.517 * * *$ \\
\hline GCDCA & -0.089 & 0.16 & $0.635 * * *$ & $0.316^{* *}$ & $0.798 * * *$ \\
\hline GDCA & $0.532 * * *$ & $0.518 * * *$ & -0.071 & -0.068 & 0.156 \\
\hline GHCA & -0.065 & -0.019 & $0.348 * * *$ & -0.047 & $0.406 * * *$ \\
\hline GCA & 0.068 & 0.152 & $0.558 * * *$ & $0.233^{*}$ & $0.758 * * *$ \\
\hline
\end{tabular}




\begin{tabular}{|c|c|c|c|c|c|}
\hline TDCA & $0.56 * * *$ & $0.59 * * *$ & 0.016 & 0.009 & $0.303 * *$ \\
\hline LCA-3S & $0.665^{* * *}$ & $0.383 * * *$ & -0.18 & 0.078 & -0.07 \\
\hline THCA & 0.04 & 0.138 & $0.337 * * *$ & -0.012 & $0.408 * * *$ \\
\hline TCA & 0.191 & $0.335 * * *$ & $0.591 * * *$ & $0.299 * *$ & $0.861 * * *$ \\
\hline
\end{tabular}

Note: BMI, body mass index; WHR, waist-hip ratio; FPG, fasting plasma glucose; 2 h PG, post-load plasma glucose; ALT, alanine aminotransferase; TG, triglyceride; HDL, high-density lipoprotein; LDL, low-density lipoprotein; TBA, total bile acids; CA, cholic acid; GCA, glycocholic acid; TCA, taurocholic acid; CDCA, chenodeoxycholic acid; GCDCA, glycochenodeoxycholic acid; TCDCA, taurochenodeoxycholic acid; DCA, deoxycholic acid; GDCA, glycodeoxycholic acid; TDCA, taurodeoxycholic acid; UDCA, ursodeoxycholic acid; GUDCA, glycoursodeoxycholic acid; TUDCA, tauroursodeoxycholic acid; LCA, lithocholic acid; GLCA, glycolithocholic acid; TLCA, taurolithocholic acid; HCA, hyocholic acid; GHCA, glycohyocholic acid; THCA, taurohyocholic acid; HDCA, hyodeoxycholic acid; GHDCA, 
glycohyodeoxycholic acid; THDCA, taurohyodeoxycholic acid; DHCA, dehydrocholic acid; GDHCA, glycodehydrocholic acid; TDHCA, taurodehydrocholic acid; 7-KLCA, 7-ketolithocholic acid; LCA-3S, lithocholic acid-3 sulfate; 3-DHCA, 3-dehydrocholic acid; 7-DHCA,

7-dehydrocholic acid; CDCA-24G, acyl CDCA-24 glucuronide. 
Table S3. Parameters of 5 models based on five NAFL-associated bile acids and clinical indices in exploration cohort.

\begin{tabular}{llll}
\hline Model & R2X(cum) & R2Y(cum) & 0.200 \\
\hline Model 1 & 0.259 & 0.040 & 0.480 \\
Model 2 & 0.523 & 0.357 & 0.300 \\
Model 3 & 0.589 & 0.485 & 0.215 \\
Model 4 & 0.284 & 0.469 & 0.216 \\
\hline
\end{tabular}

Note: Model 1, clinical factors; Model 2, all metabolites; Model 3, biomarker metabolites; Model 4, clinical factors plus all metabolites; Model 5, clinical factors plus biomarker metabolites. 
Table S4. Serum bile acid Profiles of T2DM patients with or without NAFL in exploration cohort.

\begin{tabular}{|c|c|c|c|c|c|c|}
\hline \multirow[t]{2}{*}{ Variables } & \multicolumn{2}{|c|}{ T2DM patients without NAFL(n=36) } & \multicolumn{2}{|c|}{ T2DM patients with NAFL(n=30) } & \multicolumn{2}{|l|}{$\begin{array}{l}\text { Inter-group } \\
\text { comparison } \\
(P \text {-value })\end{array}$} \\
\hline & Male & Female & Male & Female & Male $^{\mathrm{a}}$ & Female $^{\mathrm{b}}$ \\
\hline $\mathrm{N}$ & 24 & 12 & 25 & 5 & $25 v s 24$ & $5 \mathrm{vs} 12$ \\
\hline Total Unconjugated & $1152.28 \pm 180.39$ & $3562.53 \pm 1199.94$ & $1429.39 \pm 233.23$ & $2252.9 \pm 1031.43$ & 0.352 & 0.422 \\
\hline Total Glycine-conjugated & $903.40 \pm 199.24$ & $1307.26 \pm 548.47$ & $1424.09 \pm 273.35$ & $1602.38 \pm 588.51$ & 0.131 & 0.721 \\
\hline Total Taurine-conjugated & $134.72 \pm 25.25$ & $430.72 \pm 315.93$ & $357.12 \pm 92.40$ & $324.44 \pm 203.66$ & 0.028 & 0.781 \\
\hline Total CA & $382.85 \pm 73.50$ & $1108.17 \pm 392.92$ & $546.26 \pm 121.71$ & $421.18 \pm 197.56$ & 0.257 & 0.140 \\
\hline CA & $249.48 \pm 72.32$ & $813.47 \pm 280.48$ & $258.76 \pm 92.39$ & $220.82 \pm 90.11$ & 0.937 & 0.065 \\
\hline GCA & $107.48 \pm 26.21$ & $132.13 \pm 78.65$ & $207.94 \pm 62.21$ & $147.92 \pm 101.84$ & 0.146 & 0.905 \\
\hline TCA & $25.88 \pm 6.71$ & $162.57 \pm 151.86$ & $79.55 \pm 32.07$ & $52.44 \pm 42.92$ & 0.113 & 0.498 \\
\hline Total CDCA & $1027.50 \pm 142.88$ & $2120.05 \pm 555.71$ & $1389.8 \pm 256.62$ & $2250.34 \pm 701.00$ & 0.225 & 0.887 \\
\hline CDCA & $469.05 \pm 100.75$ & $1232.25 \pm 416.67$ & $463.85 \pm 98.38$ & $978.86 \pm 428.00$ & 0.971 & 0.679 \\
\hline GCDCA & $483.23 \pm 86.50$ & $703.02 \pm 283.64$ & $725.99 \pm 164.81$ & $1028.52 \pm 425.37$ & 0.200 & 0.543 \\
\hline TCDCA & $75.23 \pm 12.96^{*}$ & $184.78 \pm 116.51$ & $199.96 \pm 54.53$ & $242.96 \pm 151.34$ & 0.035 & 0.768 \\
\hline
\end{tabular}




\begin{tabular}{|c|c|c|c|c|c|c|}
\hline Total DCA & $445.35 \pm 149.30$ & $1146.47 \pm 373.06$ & $841.77 \pm 142.94$ & $211.88 \pm 92.57$ & 0.061 & 0.031 \\
\hline DCA & $213.58 \pm 37.99$ & $806.13 \pm 336.97$ & $486.41 \pm 89.05$ & $162.58 \pm 67.24$ & 0.008 & 0.086 \\
\hline GDCA & $204.10 \pm 110.52$ & $281.68 \pm 97.46$ & $295.88 \pm 65.72$ & $41.18 \pm 21.73$ & 0.480 & 0.033 \\
\hline TDCA & $27.67 \pm 11.79$ & $58.65 \pm 30.30$ & $59.48 \pm 13.12$ & $8.12 \pm 3.76$ & 0.078 & 0.125 \\
\hline Total UDCA & $297.58 \pm 85.34$ & $763.30 \pm 451.26$ & $372.20 \pm 76.81$ & $1254.3 \pm 662.41$ & 0.519 & 0.557 \\
\hline GUDCA & $90.78 \pm 23.24$ & $99.72 \pm 37.89$ & $174.56 \pm 46.12$ & $372.82 \pm 124.83$ & 0.114 & 0.093 \\
\hline TUDCA & $3.47 \pm 0.83$ & $6.03 \pm 3.71$ & $10.50 \pm 4.06$ & $16.5 \pm 8.14$ & 0.102 & 0.288 \\
\hline Total LCA & $9.58 \pm 3.54$ & $13 \pm 3.93$ & $20.63 \pm 2.85$ & $1.8 \pm 0.58$ & 0.019 & 0.016 \\
\hline $\mathrm{LCA}$ & $3.49 \pm 1.37$ & $4.07 \pm 1.99$ & $9.77 \pm 1.36$ & $0.5 \pm 0.22$ & 0.002 & 0.101 \\
\hline TLCA & $0.89 \pm 0.21$ & $1.08 \pm 0.38$ & $3.35 \pm 0.46$ & $1.02 \pm 0.27$ & 0.000 & 0.907 \\
\hline Total HCA & $51.84 \pm 8.65$ & $294.48 \pm 252.07$ & $37.80 \pm 7.60$ & $38.52 \pm 7.95$ & 0.229 & 0.332 \\
\hline $\mathrm{HCA}$ & $13.35 \pm 2.71$ & $49.06 \pm 24.69$ & $23.45 \pm 6.59$ & $25.16 \pm 8.32$ & 0.166 & 0.375 \\
\hline GHCA & $12.60 \pm 2.53$ & $82.85 \pm 76.53$ & $12.22 \pm 2.79$ & $11.66 \pm 5.23$ & 0.918 & 0.373 \\
\hline THCA & $1.58 \pm 0.25$ & $17.62 \pm 16.70$ & $2.14 \pm 0.55$ & $1.7 \pm 1.24$ & 0.371 & 0.362 \\
\hline CDCA24G & $6.58 \pm 1.59$ & $25.16 \pm 9.82$ & $73.42 \pm 22.76$ & $22.7 \pm 10.45$ & 0.007 & 0.867 \\
\hline HDCA & $1.36 \pm 0.50$ & $12.70 \pm 10.95$ & $5.48 \pm 2.22$ & $0.44 \pm 0.27$ & 0.082 & 0.287 \\
\hline
\end{tabular}




\begin{tabular}{llllllll} 
7ketoLCA & $20.81 \pm 6.35$ & $28.96 \pm 9.72$ & $13.55 \pm 2.08$ & $88.52 \pm 47.96$ & 0.287 & 0.286 \\
\hline LCA3S & $2.99 \pm 0.81 *$ & $6.10 \pm 1.46$ & $13.54 \pm 4.59$ & $1.5 \pm 1.45$ & 0.032 & 0.046 \\
\hline 7DHCA & $7.12 \pm 1.70$ & $9.70 \pm 3.17$ & $12.41 \pm 5.11$ & $51.44 \pm 32.23$ & 0.333 & 0.266 \\
\hline 3DHCA & $3.20 \pm 0.59$ & $7.00 \pm 1.87$ & $5.26 \pm 1.14$ & $11.72 \pm 4.99$ & 0.117 & 0.415 \\
\hline
\end{tabular}

Data are mean \pm SEM (nmol/l). ${ }^{a}$ means P-value between male patients T2DM with or without NAFL, ${ }^{b}$ means P-value between male patients T2DM with or without NAFL.

TBA, Total bile acids; CA, cholic acid; GCA, glycocholic acid; TCA, taurocholic acid; CDCA, chenodeoxycholic acid; GCDCA, glycochenodeoxycholic acid; TCDCA, taurochenodeoxycholicacid; DCA, deoxycholic acid; GDCA, glycodeoxycholicacid; TDCA, taurodeoxycholic acid; UDCA, ursodeoxycholic acid; GUDCA, glycoursodeoxycholic acid;

TUDCA, tauroursodeoxycholic acid; LCA, lithocholicacid; GLCA, glycolithocholic acid; TLCA, taurolithocholicacid; HCA, hyocholic acid; GHCA, glycohyocholic acid; THCA, taurohyocholic acid; HDCA, hyodeoxycholic acid; GHDCA, glycohyodeoxycholicacid; THDCA, taurohyodeoxycholic acid; DHCA, dehydrocholic acid; GDHCA, glycodehydrocholic acid;

TDHCA, taurodehydrocholic acid; 7-KLCA, 7-ketolithocholicacid; LCA-3S, lithocholic acid-3 sulfate; 3-DHCA, 3-dehydrocholic acid; 7-DHCA, 7-dehydrocholicacid; CDCA-24G, acyl

CDCA-24glucuronide. 
Table S5. Serum bile acid Profiles of T2DM patients with or without NAFL in validation cohort.

\begin{tabular}{|c|c|c|c|c|c|c|}
\hline \multirow[t]{2}{*}{ Variables } & \multicolumn{2}{|c|}{ T2DM patients without NAFL(n=20) } & \multicolumn{2}{|c|}{ T2DM patients with NAFL(n=17) } & \multicolumn{2}{|c|}{$\begin{array}{l}\text { Inter-group } \\
\text { comparison } \\
(P \text {-value) }\end{array}$} \\
\hline & Male & Female & Male & Female & Male $^{\mathrm{a}}$ & Female $^{b}$ \\
\hline $\mathrm{N}$ & 18 & 2 & 12 & 5 & $12 \mathrm{vs} 18$ & $5 v s 2$ \\
\hline Total Unconjugated & $690.73 \pm 198.47$ & $6.25 \pm 3.50$ & $902.02 \pm 176.08$ & $1479.44 \pm 311.69$ & 0.433 & l \\
\hline $\begin{array}{l}\text { Total } \\
\text { Glycine-conjugated }\end{array}$ & $523.99 \pm 52.52$ & $5.95 \pm 1.03$ & $701.81 \pm 117.94$ & $656.72 \pm 236.91$ & 0.188 & l \\
\hline $\begin{array}{l}\text { Total } \\
\text { Taurine-conjugated }\end{array}$ & $69.81 \pm 10.77$ & $73.05 \pm 43.03$ & $135.43 \pm 25.58$ & $163.52 \pm 49.70$ & 0.032 & l \\
\hline Total CA & $162.46 \pm 48.96$ & $1.35 \pm 0.04$ & $175.26 \pm 35.09$ & $392.48 \pm 113.90$ & 0.833 & l \\
\hline $\mathrm{CA}$ & $120.46 \pm 48.52$ & $187.1 \pm 117.31$ & $88.55 \pm 32.88$ & $282.04 \pm 92.80$ & 0.591 & l \\
\hline GCA & $34.28 \pm 4.61$ & $292.45 \pm 59.22$ & $64.81 \pm 14.18$ & $83.08 \pm 35.92$ & 0.061 & l \\
\hline TCA & $7.72 \pm 1.88$ & $1.95 \pm 1.38$ & $21.9 \pm 5.14$ & $27.36 \pm 8.41$ & 0.021 & l \\
\hline Total CDCA & $473.68 \pm 121.13$ & $0.50 \pm 0.35$ & $783.58 \pm 137.86$ & $1164.22 \pm 233.41$ & 0.104 & l \\
\hline
\end{tabular}




\begin{tabular}{|c|c|c|c|c|c|c|}
\hline CDCA & $185.47 \pm 95.51$ & $2.65 \pm 1.31$ & $322.87 \pm 82.63$ & $683.5 \pm 158.11$ & 0.286 & l \\
\hline GCDCA & $254.67 \pm 37.76$ & $39.45 \pm 23.23$ & $381.28 \pm 76.45$ & $369.12 \pm 116.36$ & 0.157 & I \\
\hline TCDCA & $33.55 \pm 6.83$ & $5.10 \pm 0.71$ & $79.43 \pm 15.10$ & $111.6 \pm 32.84$ & 0.014 & l \\
\hline Total DCA & $485.82 \pm 137.22$ & $44.35 \pm 29.31$ & $296.92 \pm 100.31$ & $224.2 \pm 161.43$ & 0.276 & I \\
\hline DCA & $293.23 \pm 115.76$ & $129.05 \pm 48.54$ & $176.8 \pm 72.30$ & $124.7 \pm 83.74$ & 0.401 & I \\
\hline GDCA & $167.48 \pm 31.62$ & $93.95 \pm 11.00$ & $97.73 \pm 40.21$ & $82.28 \pm 67.14$ & 0.186 & I \\
\hline TDCA & $25.11 \pm 5.62$ & $2.20 \pm 0.71$ & $22.38 \pm 7.86$ & $17.22 \pm 11.09$ & 0.780 & I \\
\hline Total UDCA & $135.95 \pm 33.27$ & $24.70 \pm 3.18$ & $450.03 \pm 131.68$ & $495.24 \pm 162.04$ & 0.039 & l \\
\hline UDCA & $81.63 \pm 22.55$ & $0.80 \pm 0.07$ & $294.14 \pm 88.22$ & $372.72 \pm 128.48$ & 0.037 & I \\
\hline GUDCA & $52.91 \pm 12.11$ & $1.05 \pm 0.74$ & $149.29 \pm 47.51$ & $117.02 \pm 37.68$ & 0.072 & I \\
\hline TUDCA & $1.41 \pm 0.40$ & $14.05 \pm 5.90$ & $6.59 \pm 2.22$ & $5.5 \pm 2.22$ & 0.041 & l \\
\hline Total LCA & $9.61 \pm 1.90$ & $7.70 \pm 0.78$ & $15.78 \pm 3.20$ & $0.92 \pm 0.40$ & 0.113 & l \\
\hline LCA & $3.41 \pm 0.91$ & $3.05 \pm 0.11$ & $8.33 \pm 1.60$ & $0.1 \pm 0.08$ & 0.015 & l \\
\hline GLCA & $5.52 \pm 1.27$ & $2.30 \pm 0.07$ & $4.17 \pm 1.56$ & $0.14 \pm 0.10$ & 0.508 & l \\
\hline TLCA & $0.68 \pm 0.09$ & $0.40 \pm 0.28$ & $3.28 \pm 0.47$ & $0.68 \pm 0.28$ & 0.000 & l \\
\hline
\end{tabular}




\begin{tabular}{lllllll} 
Total HCA & $23.38 \pm 4.92$ & $4.80 \pm 0.35$ & $16.78 \pm 3.06$ & $22.04 \pm 9.59$ & 0.264 & $/$ \\
\hline HCA & $6.54 \pm 1.93$ & $68.95 \pm 26.76$ & $11.33 \pm 2.43$ & $16.38 \pm 7.23$ & 0.137 & $/$ \\
\hline GHCA & $9.13 \pm 2.66$ & $330.20 \pm 171.76$ & $4.53 \pm 1.46$ & $5.08 \pm 2.65$ & 0.142 & $/$ \\
\hline THCA & $1.35 \pm 0.51$ & $394.10 \pm 70.99$ & $0.93 \pm 0.31$ & $0.58 \pm 0.39$ & 0.484 & $/$ \\
\hline CDCA24G & $3.07 \pm 0.62$ & $118.45 \pm 73.08$ & $32.09 \pm 7.54$ & $16.92 \pm 8.11$ & 0.003 & $/$ \\
\hline HDCA & $1.25 \pm 0.32$ & $12.15 \pm 4.28$ & $2.24 \pm 0.63$ & $1.34 \pm 0.56$ & 0.178 & $/$ \\
\hline $7 \mathrm{ketoLCA}$ & $9.84 \pm 3.71$ & $9.65 \pm 2.37$ & $13.81 \pm 5.53$ & $8.76 \pm 3.17$ & 0.557 & $/$ \\
\hline LCA3S & $2.81 \pm 0.64$ & $600.95 \pm 122.15$ & $5.27 \pm 1.69$ & $0 \pm 0$ & 9.196 & $/$ \\
\hline 7DHCA & $2.96 \pm 1.15$ & $299.35 \pm 70.04$ & $5.81 \pm 1.60$ & $9.12 \pm 2.30$ & 0.163 & $/$ \\
\hline 3DHCA & $1.3 \pm 0.32$ & $28.80 \pm 6.43$ & $3.31 \pm 1.08$ & $4.78 \pm 0.83$ & 0.099 & $/$ \\
\hline
\end{tabular}

Data are mean \pm SEM (nmol/l). ${ }^{a}$ means P-value between male patients T2DM with or without NAFL, ${ }^{b}$ it can not be compared because there were only 2 female patients T2DM without NAFL.

TBA, Total bile acids; CA, cholic acid; GCA, glycocholic acid; TCA, taurocholic acid; CDCA, chenodeoxycholic acid; GCDCA, glycochenodeoxycholic acid; TCDCA,

taurochenodeoxycholicacid; DCA, deoxycholic acid; GDCA, glycodeoxycholicacid; TDCA, taurodeoxycholic acid; UDCA, ursodeoxycholic acid; GUDCA, glycoursodeoxycholic acid; 
TUDCA, tauroursodeoxycholic acid; LCA, lithocholicacid; GLCA, glycolithocholic acid; TLCA, taurolithocholicacid; HCA, hyocholic acid; GHCA, glycohyocholic acid; THCA,

taurohyocholic acid; HDCA, hyodeoxycholic acid; GHDCA, glycohyodeoxycholicacid; THDCA, taurohyodeoxycholic acid; DHCA, dehydrocholic acid; GDHCA, glycodehydrocholic acid;

TDHCA, taurodehydrocholic acid; 7-KLCA, 7-ketolithocholicacid; LCA-3S, lithocholic acid-3 sulfate; 3-DHCA, 3-dehydrocholic acid; 7-DHCA, 7-dehydrocholicacid; CDCA-24G, acyl

CDCA-24glucuronide. 